PROCEEDINGS OF THE

AMERICAN MATHEMATICAL SOCIETY

Volume 141, Number 2, February 2013, Pages 475-480

S 0002-9939(2012)11333-4

Article electronically published on June 21, 2012

\title{
BOUNDARY-CONNECTIVITY VIA GRAPH THEORY
}

\author{
ÁDÁM TIMÁR \\ (Communicated by Richard C. Bradley)
}

\begin{abstract}
We generalize theorems of Kesten and Deuschel-Pisztora about the connectedness of the exterior boundary of a connected subset of $\mathbb{Z}^{d}$, where "connectedness" and "boundary" are understood with respect to various graphs on the vertices of $\mathbb{Z}^{d}$. These theorems are widely used in statistical physics and related areas of probability. We provide simple and elementary proofs of their results. It turns out that the proper way of viewing these questions is graph theory instead of topology.
\end{abstract}

Denote by $\mathbb{Z}^{d}$ the usual nearest-neighbor lattice on $\mathbb{Z}^{d}$, i.e., two points of $\mathbb{Z}^{d}$ are adjacent if they differ only in one coordinate, by 1 . Let $\mathbb{Z}^{d *}$ be the graph on the same vertex set and edges between every two distinct points that differ in every coordinate by at most 1 . We say that a set of vertices in $\mathbb{Z}^{d}$ is ${ }^{*}$-connected if it is connected in the graph $\mathbb{Z}^{d *}$.

In DP Deuschel and Pisztora prove that the part of the outer vertex boundary of a finite connected subgraph $C$ in $\mathbb{Z}^{d *}$ that is visible from infinity (the exterior boundary) is *-connected. Earlier, Kesten $[\mathrm{K}$ showed that the set of points in the *-boundary of a connected subgraph $C \subset \mathbb{Z}^{d *}$ that are $\mathbb{Z}^{d}$-visible from infinity is connected in $\mathbb{Z}^{d}$. Similar results were proved about the case when $C$ is in an $n \times n$ box of $\mathbb{Z}^{d}[\mathrm{DP}]$, or $\mathbb{Z}^{d *}[\mathrm{H}]$. See the second paragraphs of Theorem 3 and Theorem 4 for the precise statements.

We generalize these results about $\mathbb{Z}^{d}$ and $\mathbb{Z}^{d *}$ to a very general family of pairs of graphs; see Lemma 2, Theorem 3 and Theorem 4, Our method also gives an elementary and short alternative to the original proofs for the cubic grid case. This approach seems to be efficient to treat possible other questions about the connectedness of boundaries. Although $[\mathrm{K}$ mentions that some use of algebraic topology seems to be unavoidable, the greater generality (and simplicity) of our proof is a result of using purely graph-theoretic arguments. Also, it makes slight modifications of the results (such as considering boundaries in some subset of $\mathbb{Z}^{d}$ instead of boundaries in $\mathbb{Z}^{d}$ ) straightforward, while previously one had to go through the original proofs and make significant modifications.

In two dimensions, the use of some duality argument makes connectedness of boundaries more straightforward to prove. The lack of duality (that is, the correspondance that a cycle in one graph is a separating set in its dual) in higher

Received by the editors March 25, 2010 and, in revised form, February 21, 2011; July 1, 2011; and July 5, 2011.

2010 Mathematics Subject Classification. Primary 05C10, 05C63; Secondary 20F65, 60K35.

(C) 2012 American Mathematical Society
public domain 28 years from publication

Reverts to public domain 28 years from publication 
dimensions has been responsible for the increasing difficulty and the role of topology. Defining duality in higher dimensions led to models such as plaquette percolation, where hyperfaces ("duals" of edges) are deleted independently with some fixed probability, giving rise to random surfaces.

Theorems about connectedness of boundaries have a wide use in probability and statistical physics. To list some representatives of the many results, connectedness of the boundaries in $[\mathrm{DP}$ and $[\mathrm{K}]$ are used in the study of Ising, Potts and random cluster models $[\mathrm{Pi}, \mathrm{GG}$, first passage percolation $[\mathrm{K}$, Bernoulli percolation $\mathrm{KZ}$, $\mathrm{AP}$ and random walks on percolation clusters $\mathrm{Pe}$, entanglement percolation $[\mathrm{GH}$, greedy lattice animals $[\mathrm{H}]$. Understanding connectedness of boundaries is an essential part for the use of Peierls estimates, and for proving the existence of phase transitions. The fundamental role of these results in many statistical physics arguments makes it important to understand these issues properly. Our generalizations may help extend some of these results to graphs beyond $\mathbb{Z}^{d}$. This was the case in the simplification of the results of $[\mathrm{BB}]$ in $[\mathrm{T}]$, and the main lemma in the latter is the starting point of the current paper (see Lemma 1). Even for the cases where $\mathbb{Z}^{d}$ is considered, the use of elementary graph-theoretic arguments instead of topology adds a lot of flexibility and makes the proofs more accessible.

The graphs we consider can be finite or infinite, but we always assume that they are locally finite (that is, every vertex has finite degree). Given a subgraph $H$ of a graph $G$, the inner boundary of $H$ in $G$ is the set of vertices in $H$ that are adjacent to some vertex in $G \backslash H$. Similarly, the outer boundary of $H$ in $G$ is the set of vertices in $G \backslash H$ that are adjacent to some vertex in $H$. If $G$ is infinite and $H$ is finite, the exterior part of a boundary (of either type) is the set of vertices in the boundary that are starting points of some infinite path with no interior vertex in $H$. The boundaries we consider are always taken to be outer boundaries, but our arguments would apply just as well for inner boundaries. By a separating set we always understand a separating set of vertices. In this paper addition is always understood modulo 2, and this is how we define the sums of sets of edges (regarded as vectors over the 2-element field). In particular, this defines the generation of cycles by other cycles. Let the cycle space of a graph $G$ be the set of all finite subgraphs such that every vertex has an even degree. It is well known that the cycle space is generated by the set of cycles.

For an arbitrary graph $G$, let $\operatorname{Ends}(G)$ be the the set of ends in $G$, where an end is an equivalence class of infinite simple paths, two being equivalent if they can be connected by infinitely many pairwise disjoint paths. So, $\operatorname{Ends}(G)=\emptyset$ iff $G$ is finite, and for $G=\mathbb{Z}^{d}$ we have $|\operatorname{Ends}(G)|=1$. A path from an end $x$ (or, between an end and a vertex $y$ ) is some path in the equivalence class that defines $x$ (and starts from $y$ respectively). A path between two ends $x, y$ is a bi-infinite path $P$ such that for any $v \in P, P \backslash v$ consists of a path that belongs to $x$ and a path that belongs to $y$. A separating set between $x \in V(G) \cup \operatorname{Ends}(G)$ and a $y \in V(G) \cup \operatorname{Ends}(G)$ is a subset of $V(G)$ that every path between $x$ and $y$ intersects. A separating set of edges between $x \in V(G) \cup \operatorname{Ends}(G)$ and a $y \in V(G) \cup \operatorname{Ends}(G)$ is a subset of $E(G)$ that every path between $x$ and $y$ intersects. An important property of minimal separating sets of edges is that they always split a connected graph into two components (this may not be true for separating sets of vertices). 
Given some graph $G$ and a graph $H$ containing $G$, we say that a cycle $C$ in $G$ is chordal in $H$ if any two points in $C$ are adjacent in $H$. If $\mathcal{C}$ is a set of cycles in $G$, we say that $\mathcal{C}$ is chordal in $H$ if every cycle in $\mathcal{C}$ is chordal in $H$.

The next lemma is the key to our proofs. Similar and slightly weaker versions are in $[\mathrm{BB}$ and $\mathrm{T}$.

Lemma 1. Let $G$ be some graph and $\Pi$ be a minimal separating set of edges between two points $x, y \in G \cup \operatorname{Ends}(G)$. Let $\mathcal{C}$ be a set of cycles that generate the cycle space of $G$. Then for any partition $\left(\Pi_{1}, \Pi_{2}\right)$ of $\Pi$, there is some cycle $O \in \mathcal{C}$ that intersects both $\Pi_{1}$ and $\Pi_{2}$. There is also an $O$ with the above property and such that $\left|O \cap \Pi_{2}\right|$ is odd.

Proof. If $x$ (or $y$ ) is an end, define $x^{\prime}\left(y^{\prime}\right)$ to be a vertex such that there is a path between $x$ and $x^{\prime}\left(y\right.$ and $\left.y^{\prime}\right)$ in $G \backslash \Pi$. Otherwise let $x^{\prime}:=x\left(y^{\prime}:=y\right)$. Choose paths $P_{i}$ between $x^{\prime}$ and $y^{\prime}, i=1,2$, such that $P_{i}$ does not intersect $\Pi_{3-i}$. Such paths exist by the minimality of $\Pi$. There is a subset $A \subset \mathcal{C}$ such that

$$
P_{1}+P_{2}=\sum_{C \in A} C .
$$

Let $A_{1} \subset A$ be the set of those cycles that intersect $\Pi_{1}$ and $A_{2}:=A \backslash A_{1}$. The previous equation can be written as

$$
P_{1}+\sum_{C \in A_{1}} C=P_{2}+\sum_{C \in A_{2}} C .
$$

The right-hand side here does not intersect $\Pi_{1}$, so it has to intersect $\Pi_{2}$ (since $x^{\prime}$ and $y^{\prime}$ are the only vertices with odd degree in $P_{2}+\sum_{C \in A_{2}} C$, so they belong to the same component of it). Furthermore, $P_{2}$ contains an odd number of elements from $\Pi_{2}$, and every cycle in $A_{2}$ contains an even number of elements from $\Pi_{2}$. Thus the total number of elements of $\Pi_{2}$ in the sum on the right side is odd. We conclude that the left side (regarded as a subgraph of $G$ ) has to contain some cycle $O$ that intersects $\Pi_{2}$ in an odd number of edges (since $P_{1}$ does not intersect $\Pi_{2}$ ), and $O \cap \Pi_{1} \neq \emptyset$ too, by the definition of $A_{1}$.

For a subgraph $C$ of $G$, and $x \in V(G) \cup \operatorname{Ends}(G)$, the outer boundary of $C$ visible from $x$ is $\partial_{\operatorname{vis}(x)}(C):=\{y \in V(G): y$ is adjacent to some point in $C$, and there is a path between $x$ and $y$ disjoint from $C$ \}. When there are two graphs, $G$ and $G^{\prime}$ on the same vertex set, we will also use $\partial_{\text {vis }_{G}(x)}^{G^{\prime}}(C):=\{y \in V(G): y$ is $G^{\prime}$-adjacent to some point in $C$, and there is a $G$-path between $x$ and $y$ disjoint from $C$ \}. Hence $\partial_{\operatorname{vis}_{G}(x)}^{G}(C)=\partial_{\operatorname{vis}(x)}(C)$.

Let $B_{n}$ denote the box induced by $\{1, \ldots, n\}^{d}$ in $\mathbb{Z}^{d}$. By a basic 4 -cycle of $\mathbb{Z}^{d}$ we mean the 4 -cycle surrounding some 2 -face in a unit cube in $\mathbb{Z}^{d}$. Note that the cycle space of $\mathbb{Z}^{d}$ has a generating set of basic 4-cycles: think about $\mathbb{Z}^{d}$ as a Cayley graph for the free Abelian group. Then the set of basic 4-cycles is the set of all conjugates of the pairwise commutators of the generating elements, whose products generate any word equal to the identity - and cycles of $\mathbb{Z}^{d}$ correspond to such words.

The *-connectedness of the $\mathbb{Z}^{d}$-boundary of a finite connected set in $\mathbb{Z}^{d *}$ is shown in $\mathrm{DP}$. We prove a weaker statement here, assuming that the connected set is from $\mathbb{Z}^{d}$. We will prove the (generalization of) the original version later in Theorem 3 , with more assumptions on the underlying graphs. 
Lemma 2. Let $G$ be a graph and $G^{+}$be a graph that contains $G$. Suppose that there is a generating set $\Delta_{G}$ for the cycle space of $G$ that is chordal in $G^{+}$. Then for any connected subset $C$ of $G$ and any $x \in(V(G) \cup \operatorname{Ends}(G)) \backslash C$, the set $\partial_{\mathrm{vis}_{G}(x)}^{G}(C)$ induces a connected graph in $G^{+}$.

In particular, any finite connected subset of $\mathbb{Z}^{d}$ has a ${ }^{*}$-connected exterior $\mathbb{Z}^{d}$ boundary, and if $C \subset B_{n}$, the outer $\mathbb{Z}^{d}$-boundary of $C$ in any component of $B_{n} \backslash C$ is *-connected.

Proof. Let $\Pi:=\left\{\{u, v\} \in E(G): u \in C, v \in \partial_{\mathrm{vis}_{G}(x)}^{G}(C)\right\}$. Then $\Pi$ is a minimal separating set of edges in $G$ between $C$ and $x$. because for every edge $e \in \Pi$ there is a $G \backslash \Pi$-path from $x$ to the endpoint of $e$ in $\partial_{\text {vis }_{G}(x)}^{G}(C)$, and appending $e$ to this path we get a path from $C$ to $x$ that intersects $\Pi$ only in $e$.

Let $\partial_{\mathrm{vis}_{G}(x)}^{G}(C)=S_{1} \cup S_{2}$ be an arbitrary partition. Further, partition $\Pi$ to sets $\Pi_{i}:=\left\{\{x, y\} \in E(G): x \in C, y \in S_{i}\right\}, i=1,2$. By Lemma 1, there is a cycle $O \in \Delta_{G}$ such that $O \cap \Pi_{1} \neq \emptyset$ and $O \cap \Pi_{2} \neq \emptyset$. Take an edge from each of these intersections and consider their endpoints in $C$. These are adjacent in $G^{+}$, since $O$ is chordal, and hence the $G^{+}$-distance of $S_{1}$ and $S_{2}$ is 1 . Since the partition to $S_{1}$ and $S_{2}$ was arbitrary, we conclude that $\partial_{\mathrm{vis}_{G}(x)}^{G}(C)$ is $G^{+}$-connected.

For Lemma 2 to hold with a $C$ that is $G^{*}$-connected but not necessarily $G$ connected (which is the form of the result in $[\mathrm{DP}$ ), we need some extra assumptions on the cycle space. Without those, the conclusion of Lemma 2 need not hold, as shown by $G=\mathbb{Z}^{2}, G^{+}=\mathbb{Z}^{2 *} \cup\{\{u, v\}\}$, where $\{u, v\}$ is an edge with endpoints at distance 10 in $\mathbb{Z}^{2}$, and we choose $C$ to be the $G^{+}$-connected set induced by the 2-neighborhoods of $x$ and $y$ in $G^{+}$.

Theorem 3. Let $G^{+}$be a connected graph and $G$ a connected subgraph of $G^{+}$. Suppose that there is a generating set $\Delta_{G}$ for the cycle space of $G$ that is chordal in $G^{+}$, and that for every edge $e \in G^{+}$there is a cycle $O_{e}$ in $G^{+}$such that $O_{e} \backslash e \subset G$, and $O_{e}$ is chordal in $G^{+}$. Let $C$ be a connected subgraph of $G^{+}$, and $x \in(V(G) \cup$ $\left.\operatorname{Ends}\left(G^{+}\right)\right) \backslash C$. Then $\partial_{\text {vis }_{G}(x)}^{G}(C)$ is connected in $G^{+}$.

In particular, any finite ${ }^{*}$-connected subset of $\mathbb{Z}^{d}$ has a ${ }^{*}$-connected exterior $\mathbb{Z}^{d}$ boundary, and if $C \subset B_{n}$, the outer $\mathbb{Z}^{d}$-boundary of $C$ in any component of $B_{n} \backslash C$ is * - connected.

Note that Theorem 3 is stronger than the one in $\overline{D P}$ even in the $\mathbb{Z}^{d}$ case: it implies that the boundary of a connected subset of $\mathbb{Z}^{d}$ is connected in the graph $\mathbb{Z}^{d} \cup\{$ edges connecting two points of some basic 4-cycle\}, which does not follow from the topological proof in $[\mathrm{DP}]$. (This strengthening was first shown $\left(\right.$ for $\mathbb{Z}^{d}$ ) in GG.

Proof of Theorem 3. Define $S:=\partial_{\mathrm{vis}_{G}(x)}^{G}(C)$. Let $\Pi:=\{\{x, y\} \in E(G): x \in$ $C, y \in S\}$ and $H$ be a graph with $V(H)=V(G)$ and $E(H)=\left.G^{+}\right|_{C} \cup E(G)$ (here by $\left.G^{+}\right|_{C}$ we denote the subgraph of $G^{+}$induced by $C$ ). Then $\Pi$ is a separating set of edges between $C$ and $x$ in $H$, and it is a minimal separating set of edges, because for every edge $e \in \Pi$ there is a path in $G \backslash \Pi$ from $x$ to the endpoint of $e$ in $S$, and appending $e$ to this path we get an $H$-path from $C$ to $x$ that intersects $\Pi$ only in $e$.

Let $\Delta$ be a generating set for the cycles of $H$, consisting of cycles that are chordal in $G^{+}$. We are going to show the existence of such a $\Delta$. By our assumptions $\mathcal{H}:=\left\{O_{e}: e \in H \backslash G\right\} \cup \Delta_{G}$ consists of cycles that are chordal in $G^{+}$. On the 
other hand, any cycle $U$ in $H$ is generated by $\mathcal{H}$, because $U+\sum_{e \in U \backslash G} O_{e}$ is a 2-regular graph in $G$, and hence it is generated by $\Delta_{G}$.

Let $S=S_{1} \cup S_{2}$ be an arbitrary partition. Further, partition $\Pi$ to sets $\Pi_{i}:=$ $\left\{\{x, y\} \in E(G): x \in C, y \in S_{i}\right\}, i=1,2$. By Lemma 1 there is an $O \in \Delta$ with $O \cap \Pi_{1} \neq \emptyset$ and $O \cap \Pi_{2} \neq \emptyset$. Since $O$ is chordal in $G^{+}$, we obtain that the $G^{+}$-distance between $S_{1}$ and $S_{2}$ is 1 . Since their choice was arbitrary, $S$ necessarily induces a connected graph in $G^{+}$.

The case $G=\mathbb{Z}^{d}$ follows by choosing $\Delta$ to be a generating set of basic 4-cycles. For an edge $e \in \mathbb{Z}^{d *}$, let $O_{e}$ be a cycle such that $O_{e} \backslash e$ only has edges from a unit cube that contains $e$.

The $\mathbb{Z}^{d}$ version of the following theorem is due to Kesten. Its proof in $[\mathrm{K}]$ takes a section, with references to results from algebraic topology. The similiar statement for the box of $\mathbb{Z}^{d}$ as $G$ was proved in $[\mathrm{H}$ ( and it did not follow automatically from Kesten's result).

Theorem 4. Let $G^{+}$be a connected graph and $G$ a connected subgraph of $G^{+}$. Suppose that there is a generating set $\Delta_{G}$ for the cycle space of $G$ that is chordal in $G^{+}$, and that for every edge $e \in G^{+}$there is a cycle $O_{e}$ in $G^{+}$such that $O_{e} \backslash e \subset G$, and $O_{e}$ is chordal in $G^{+}$. Let $C$ be a connected subgraph of $G^{+}$and $x \in(V(G) \cup$ $\left.\operatorname{Ends}\left(G^{+}\right)\right) \backslash C$. Then $\partial_{\mathrm{vis}_{G}(x)}^{G^{+}}(C)$ is connected in $G$.

In particular, if $C \subset \mathbb{Z}^{d}$ is finite and ${ }^{*}$-connected, then the subset of its exterior outer boundary in $\mathbb{Z}^{d *}$ that is accessible by an infinite path in $\mathbb{Z}^{d} \backslash C$ is $\mathbb{Z}^{d}$-connected. If $C$ is a subset of $B_{n}, x \in B_{n} \backslash C$, then $\partial_{\mathrm{vis}_{\mathbb{Z}^{d}}(x)}^{\mathbb{Z}^{d *}}(C)$ is $\mathbb{Z}^{d}$-connected.

The first half of the proof is very similar to that of Theorem 3 . The only difference between the proofs is that we have to define the auxiliary graphs $H$ slightly differently and that we need some more arguments in Theorem 4 for the conclusion.

Proof of Theorem 4. Define $S:=\partial_{\text {vis }_{G}(x)}^{G^{+}}(C)$. Let $\Pi:=\left\{\{x, y\} \in E\left(G^{+}\right): x \in\right.$ $C, y \in S\}$ and $H$ be a graph with $V(H)=V(G)$ and $E(H)=\left.G^{+}\right|_{C} \cup E(G) \cup \Pi$. Similarly to the proof of Theorem 3 , $\Pi$ is a minimal separating set of edges between $C$ and $x$ in $H$, and there exists a $\Delta$ generating set for the cycles of $H$, consisting of cycles that are chordal in $G^{+}$.

Let $S=S_{1} \cup S_{2}$ be an arbitrary partition. Further, partition $\Pi$ to sets $\Pi_{i}:=$ $\left\{\{x, y\} \in E(G): x \in C, y \in S_{i}\right\}, i=1,2$. By Lemma 1 there is an $O \in \Delta$ with $O \cap \Pi_{1} \neq \emptyset$ and $\left|O \cap \Pi_{2}\right|$ odd.

Suppose first that $O$ contains some vertex $v$ not in $C \cup S$. Let $C_{G^{+}}(x)$ be the component of $x$ in $G^{+} \backslash \Pi$, and let $C_{H}(x)$ be the component of $x$ in $H \backslash \Pi$. By the chordality of $O$, there is an edge between some vertex $w \in O \cap C$ and $v$. If $v \in C_{H}(x)$, then this would imply $v \in S$, contradicting the assumption on $v$. So suppose $v \notin C_{H}(x)$. But the cycle $O_{\{v, w\}}$ is such that every edge of it different from $\{v, w\}$ is in $G$. In particular, there is a $G$-path from $v$ to $S$ : this path goes from $v$ to the element $u$ of $S$ that is the neighbor of $w$ inside $O$, and appending this path to the path from $u$ to $x$ gives that $v$ should be in $C_{H}(x)$, a contradiction.

Hence $V(O) \subset C \cup S$. Call a set of vertices $B \subset S_{2}$ in $O$ a block if $B$ induces a connected subgraph in $O$ (i.e., a subpath) and it is maximal with this property. Let $I$ be the set of edges in $O$ that have exactly one endpoint in $B$. It is clear by the definition that $O \cap \Pi_{2} \subset I$ and that $|I|$ is even, since every block contributes 
two edges to it. If there is an edge $e$ in $I$ such that the other endpoint of $e$ is in $S_{1}$, then the proof is finished: $S_{1}$ and $S_{2}$ have distance 1 in $H$ (and hence in $G$, since $\left.\left.H\right|_{S}=\left.G\right|_{S}\right)$. So suppose not: every $e \in I$ has the form $e=\{x, y\}$ with $x \in S_{2}$, $y \in V(O) \backslash S \subset C$ (using the assumption $V(O) \subset C \cup S$ ). That is, $I=O \cap \Pi_{2}$. But by the fact that $I$ has an even number of elements, this would contradict the choice of $O$ (that $\left|O \cap \Pi_{2}\right|$ is odd).

The $\mathbb{Z}^{d}$ case follows by the same argument as at the end of the proof of Theorem 3 ,

Remark 5. The proofs of Theorem 4 and Theorem 3 show that the conditions on the cycle spaces of $G$ and $G^{+}$can be weakened or stated differently: the only important thing is that we can generate the cycle space of $H$ by cycles that are chordal in $G^{+}$.

\section{ACKNOWLEDGEMENTS}

The author is very grateful to Gábor Pete for drawing his attention to the subject and for helpful conversations. He also thanks Geoffrey Grimmett for his comments on the manuscript. Finally, he is indebted to an anonymous referee for corrections and suggestions, which led to further simplifications of the proofs.

\section{REFERENCES}

[AP] Antal, P. and Pisztora, Á. (1996) On the chemical distance for supercritical Bernoulli percolation, Ann. Probab. 24, no. 2, 1036-1048. MR1404543 (98b:60168)

[BB] Babson, E. and Benjamini, I. (1999) Cut sets and normed cohomology with applications to percolation, Proc. Amer. Math. Soc. 127, 589-597. MR1622785 (99g:05119)

[DP] Deuschel, J. and Pisztora, Á. (1996) Surface order large deviations for high-density percolation, Prob. Theory and Related Fields 104, 467-482. MR1384041 (97d:60053)

[GG] Gielis, G. and Grimmett, G. (2002) Rigidity of the interface in percolation and randomcluster models, J. Stat. Phys. 109, 1-37. MR.1927913 (2003h:82037)

[H] Hammond, A. (2006) Greedy lattice animals: geometry and criticality, Ann. Probab. 34, no. 2, 593-637. MR:2223953 (2007b:60238)

[GH] Grimmett, G. and Holroyd, A. (2000) Entanglement in percolation, Proc. London Math. Soc. (3) 81, no. 2, 485-512. MR1770617(2001j:60185)

[K] Kesten, H. (1986) Aspects of first-passage percolation, in École d'été de probabilité de SaintFlour XIV, Lecture Notes in Math. 1180, Springer-Verlag, 125-264. MR876084 (88h:60201)

[KZ] Kesten, H. and Zhang, Y. (1990) The probability of a large finite cluster in supercritical Bernoulli percolation, Ann. Probab. 18, no. 2, 537-555. MR1055419 (91i:60278)

[Pe] Pete, G. (2008) A note on percolation on $\mathbb{Z}^{d}$ : isoperimetric profile via exponential cluster repulsion, Elect. Comm. Probab. 13, 377-392. MR2415145 (2010b:60272)

[Pi] Pisztora, Á. (1996) Surface order large deviations for Ising, Potts and percolation models, Probability Theory Rel. Fields 104, 427-466. MR1384040(97d:82016)

[T] Timár, Á. (2007) Cutsets in infinite graphs, Combin. Probab. and Comp. 16, issue 1, 159166. MR 2286517 (2007j:05102)

Hausdorff Center for Mathematics, Universität Bonn, D-53115 Bonn, Germany

E-mail address: adam.timar@hcm.uni-bonn.de 